October, 1995

\title{
Exactly solvable extended Hubbard model
}

\author{
D. F. Wang \\ Institut de Physique Théorique \\ Ecole Polytechnique Fédérale de Lausanne \\ PHB-Ecublens, CH-1015 Lausanne, Switzerland
}

\begin{abstract}
In this work, we introduce long range version of the extended Hubbard model. The system is defined on a non-uniform lattice. We show that the system is integrable. The ground state, the ground state energy, and the energy spectrum are also found for the system. Another long range version of the extended Hubbard model is also introduced on a uniform lattice, and this system is proven to be integrable.
\end{abstract}

PACS number: 71.30.+h, 05.30.-d, 74.65+n, 75.10.Jm

Typeset Using REVTEX 
In recent years, there have been considerable interests in low dimensional electronic systems of low dimensions. Systems of this type may exhibit interesting novel physics, due to low dimensionality and strong correlation. Anderson has suggested that the 2D one band Hubbard model should explain the basic physics of the high temperature superconductivity [1]. It is suggested that the normal state of the new cuperate oxide superconductors may share the feature (Luttinger-liquid-like) of 1D interacting electron gas [1,2]. This physical motivation has initiated lots of recent activities in one dimensional electronic models.

In one dimension, the Hubbard model was exactly solved by Lieb and Wu [3]. With the $S O(4)$ symmetry due to Yang and Zhang [四, 5], the completeness of the eigenstates in this structure has been discussed [6]. More recently, an extended Hubbard model was introduced by several authors in general $d$ dimensions [6]. By using $\eta$-paring mechanism, they construct eigenstates of the system, with off-diagonal-long-range order [7]. Particularly, its one dimensional version was solved exactly with Bethe-ansatz [7]. In this work, we introduce the long range version of the extended Hubbard model in one dimension. We show that the system is completely integrable, with explicit construction of infinite constants of motion. The ground state, the energy spectrum are found for the system.

Let us consider a one dimension lattice of sites $L$. The positions of the sites are given by the roots of the Hermite polynomial $H_{L}(x)$. The roots of the Hermite polynomial $r_{1}, r_{2}, \cdots, r_{L}$ are all real and distinct, and this non-uniform lattice is thus well-defined. Consider the following Hamiltonian:

$$
H=-\frac{1}{2} \sum_{1 \leq i \neq j \leq L} J_{i j} \Pi_{i j},
$$

where $J_{i j}=1 /\left(r_{i}-r_{j}\right)^{2}$, and the permutation operator $\Pi_{i j}$ is given by

$$
\begin{aligned}
\Pi_{i j}= & c_{j \uparrow}^{\dagger} c_{i \uparrow}\left(1-n_{i \downarrow}-n_{j \downarrow}\right)+c_{i \uparrow}^{\dagger} c_{j \uparrow}\left(1-n_{i \downarrow}-n_{j \downarrow}\right) \\
& +c_{j \downarrow}^{\dagger} c_{i \downarrow}\left(1-n_{i \uparrow}-n_{j \uparrow}\right)+c_{i \downarrow}^{\dagger} c_{j \downarrow}\left(1-n_{i \uparrow}-n_{j \uparrow}\right) \\
& +\frac{1}{2}\left(n_{i}-1\right)\left(n_{j}-1\right)+c_{i \uparrow}^{\dagger} c_{i \downarrow}^{\dagger} c_{j \downarrow} c_{j \uparrow}+c_{i \downarrow} c_{i \uparrow} c_{j \uparrow}^{\dagger} c_{j \downarrow}^{\dagger} \\
& -\frac{1}{2}\left(n_{i \uparrow}-n_{i \downarrow}\right)\left(n_{j \uparrow}-n_{j \downarrow}\right)
\end{aligned}
$$




$$
\begin{aligned}
& -c_{i \downarrow}^{\dagger} c_{i \uparrow} c_{j \uparrow}^{\dagger} c_{j \downarrow}-c_{i \uparrow}^{\dagger} c_{i \downarrow} c_{j \downarrow}^{\dagger} c_{j \uparrow} \\
& +\left(n_{i \uparrow}-1 / 2\right)\left(n_{i \downarrow}-1 / 2\right)+\left(n_{j \uparrow}-1 / 2\right)\left(n_{j \downarrow}-1 / 2\right),
\end{aligned}
$$

where $c_{i \sigma}$ and $c_{i \sigma}^{\dagger}$ are electron annihilation and creation operators at site $i$ with spin $\sigma=\uparrow, \downarrow$. The electron number operators are given by $n_{i \uparrow}=c_{i \uparrow}^{\dagger} c_{i \uparrow}, n_{i \downarrow}=c_{i \downarrow}^{\dagger} c_{i \downarrow}, n_{i}=n_{i \uparrow}+n_{i \downarrow}$. If one defines the Hamiltonian on a uniform lattice and the coupling parameter $J_{i j}=\delta_{1,|i-j|}$, the system becomes the extended Hubbard model studied by Ebler, Korepin and Schoutens [7].

For the long range extended Hubbard model defined on this non-uniform lattice, we'll present its ground state, the energy spectrum and the proof of integrability in the following. At each site $i$, one can use the standard slave boson representation:

$$
\begin{aligned}
& |\downarrow \uparrow><0|=p_{i}^{\dagger} b_{i} \\
& |\downarrow \uparrow><\sigma|=p_{i}^{\dagger} f_{i \sigma} \\
& |\sigma><\tau|=f_{i \sigma}^{\dagger} f_{i \tau} \\
& |\sigma><0|=f_{i \sigma}^{\dagger} b_{i} \\
& |0><\sigma|=b_{i}^{\dagger} f_{i \sigma},
\end{aligned}
$$

where $\left|\downarrow \uparrow>=c_{i \downarrow}^{\dagger} c_{i \uparrow}^{\dagger}\right| 0>,\left|\sigma>=c_{i \sigma}^{\dagger}\right| 0>$, at site $i$. For these relations to hold, the pair operators $p$ and the hole operators $b$ are bosonic, while the operators $f$ are fermionic:

$$
\begin{aligned}
& {\left[b_{i}, b_{j}^{\dagger}\right]=\delta_{i j}} \\
& {\left[p_{i}, p_{j}^{\dagger}\right]=\delta_{i j}} \\
& {\left[f_{i \sigma}, f_{j \tau}^{\dagger}\right]_{+}=\delta_{i j} \delta_{\sigma \tau},}
\end{aligned}
$$

with the constrain that at any site $i$ there is always one particle, i.e., $\sum_{\sigma=\uparrow, \downarrow} f_{i \sigma}^{\dagger} f_{i \sigma}+b_{i}^{\dagger} b_{i}+$ $p_{i}^{\dagger} p_{i}=1$. With the slave boson representation, a state vector can be written as below:

$$
\begin{gathered}
\mid \phi>=\sum_{\{x\},\{y\},\{z\}} \phi\left(x_{1}, x_{2}, \cdots, x_{A}\left|y_{1} \sigma_{1}, y_{2} \sigma_{2}, \cdots, y_{M} \sigma_{M}\right| z_{1}, z_{2}, \cdots, z_{Q}\right) \cdot \\
\cdot \prod_{\alpha=1}^{A} p_{x_{\alpha}}^{\dagger} \prod_{l=1}^{M} f_{y_{l} \sigma_{l}}^{\dagger} \prod_{i=1}^{Q} b_{z_{i}}^{\dagger} \mid 0>
\end{gathered}
$$


where the wavefunction $\phi$ is symmetric when exchanging $\{x\}$ or $\{z\}$ respectively, while anti-symmetric when exchanging $y_{i} \sigma_{i}$ and $y_{j} \sigma_{j}$. In the following, we use $\left(q_{1}, q_{2}, \cdots, q_{L}\right)=$ $\left(x_{1}, \cdots, x_{A}\left|y_{1}, \cdots, y_{M}\right| z_{1}, \cdots, z_{Q}\right)$.

With the Hamiltonian $H$ given by Eq. (1), the number of the electron pairs, the number of holes, the number of the sites single-occupied by up-spin electrons, the number of the sites single-occupied by the down-spin electrons, are all conserved quantities. Let us denote them by the following notations:

$$
\begin{aligned}
& A=\sum_{i=1}^{L} n_{i \downarrow} n_{i \uparrow} \\
& M_{\uparrow}=\sum_{i=1}^{L} n_{i \uparrow}-A \\
& M_{\downarrow}=\sum_{i=1}^{L} n_{i \downarrow}-A \\
& Q=L-M_{\uparrow}-M_{\downarrow}-A \\
& M=M_{\uparrow}+M_{\downarrow} .
\end{aligned}
$$

In the following, we work in the Hilbert space of fixed $A, M_{\uparrow}, M_{\downarrow}$ and $Q$.

Using the amplitude $\phi$ defined by the Eq. (5), the eigenenergy equation can thus reduce to

$$
-\frac{1}{2} \sum_{1 \leq i \neq j \leq L} \frac{1}{\left(q_{i}-q_{j}\right)^{2}} M_{i j} \phi(\{q\})=E \phi(\{q\}),
$$

where the operator $M_{i j}$ permutes the coordinates $q_{i}$ and $q_{j}$. The Hamiltonian $H=$ $-\frac{1}{2} \sum_{1 \leq i \neq j \leq L}\left(q_{i}-q_{j}\right)^{-2} M_{i j}$ commutes with the infinite number of simultaneous constants of motion, i.e., $\left[H, I_{n}\right]=0,\left[I_{n}, I_{m}\right]=0$, with $n, m=1,2, \cdots, \infty$, where $I_{n}=\sum_{i=1}^{L} h_{i}^{n}, h_{i}=a_{i}^{\dagger} a_{i}$, with $a_{j}^{\dagger}=\sum_{k(\neq j)=1}^{L} i\left(q_{j}-q_{k}\right)^{-1} M_{j k}+i q_{j}, a_{i}=\left(a^{\dagger}\right)^{\dagger}$, with $j=1,2, \cdots, L$ [9]8]. This shows that this long range version of extended Hubbard model is also integrable. When there are no electron pairs, this system reduces to the long range t-J model studied before [8,[10]. When there are no electron pairs and no holes, the system becomes a previous spin chain [9]. 
We first construct the ground state wavefunction in the general subspace of fixed non-zero $A, M_{\uparrow}, M_{\downarrow}$ and $Q$. Let us look at the following Jastrow product wavefunction:

$$
\begin{array}{r}
\phi\left(x_{1}, x_{2}, \cdots, x_{A}\left|y_{1} \sigma_{1}, y_{2} \sigma_{2}, \cdots, y_{M} \sigma_{M}\right| z_{1}, z_{2}, \cdots, z_{Q}\right) \\
=\prod_{1 \leq i<j \leq M}\left(y_{i}-y_{j}\right)^{\delta_{\sigma_{i}} \sigma_{j}} e^{i \frac{\pi}{2} \operatorname{sgn}\left(\sigma_{i}-\sigma_{j}\right)}
\end{array}
$$

This wavefunction is anticipated to be the ground state of the system in the subspace of fixed $A, M_{\uparrow}, M_{\downarrow}$ and $Q$. With previous experience [10], one may show that this wavefunction is indeed an eigenstate of the Hamiltonian, with the following eigenenergy

$$
E_{0}=-\frac{1}{4} L(L-1)+\frac{1}{2} M_{\uparrow}\left(M_{\uparrow}-1\right)+\frac{1}{2} M_{\downarrow}\left(M_{\downarrow}-1\right) .
$$

For this Hamiltonian, one may show that $a_{j}^{\dagger}$ and $a_{j}$ are raising or lowering operators defined on this lattice, $\left[a_{j}^{\dagger}, H\right]=-a_{j}^{\dagger},\left[a_{j}, H\right]=a_{j}$. One may simply prove following identities:

$$
\begin{aligned}
& a_{i} \phi=0 ; \quad i=1,2, \cdots, M \\
& a_{i} \phi=0 ; \quad i=A+M+1, A+M+2, \cdots, L .
\end{aligned}
$$

Therefore, one can see that

$$
\begin{aligned}
& \left(\sum_{i=1}^{M} a_{i}\right) \phi=0, \\
& \left(\sum_{i=A+M+1}^{L} a_{i}\right) \phi=0 .
\end{aligned}
$$

Furthermore, one can show that

$$
\left(\sum_{i=1}^{M} a_{i+A} \sigma_{i}^{z}\right) \phi=0 .
$$

The above three identities show the impossibilities of constructing non-zero eigenstates of lower energy than $E_{0}$ with the annihilation operators. One may regard this as a partial support for our idea that $\phi$ is the ground state in the subspace of fixed $A, M_{\uparrow}, M_{\downarrow}, Q$. For a more complete confirmation, further work is needed in this case.

The full energy spectra of the system consists of energy levels equal-spaced, 


$$
E(s)=E_{0}+s
$$

where $s=0,1,2, \cdots$. For a lattice of finite size $L$, there is an upper bound on the value of $s$, as the Hilbert space of the system is finite. There are several ways of constructing excited states. The first way is to excite those electron pairs:

$$
\left|n_{1}, n_{2}, \cdots, n_{A}>=\sum_{P} \prod_{i=1}^{A}\left(a_{i}^{\dagger}\right)^{n_{P_{i}}}\right| \phi>
$$

where $n_{1}, n_{2}, \cdots, n_{A}$ are integers or zero, the summation $P$ is over all possible permutations. Under the operation of the creation operators, any states constructed this way, if not vanishing, will be the eigenstates of the Hamiltonian with eigenenergy

$$
E_{1}=E_{0}+\left(n_{1}+n_{2}+\cdots+n_{A}\right) .
$$

The second way to create excitations is to excite the holes of the system:

$$
\left|m_{1}, m_{2}, \cdots, m_{Q}>=\sum_{P}\left(a_{A+M+1}^{\dagger}\right)^{m_{P_{1}}}\left(a_{A+M+2}^{\dagger}\right)^{m_{P_{2}}} \cdots\left(a_{A+M+Q}^{\dagger}\right)^{m_{P_{Q}}}\right| \phi>
$$

with the eigenenergies given by

$$
E_{2}=E_{0}+\left(m_{1}+m_{2}+\cdots+m_{Q}\right),
$$

if these state vectors are not zero. Finally, one may create excitations by exciting the electrons on the sites single-occupied. These excited states may be written as

$$
\left.\mid \phi_{3}>=\sum_{P}\left(S_{1}\left(\nu_{1}\right)^{\dagger}\right)^{n_{P_{1}}}\left(S_{2}\left(\nu_{2}\right)\right)^{\dagger}\right)^{n_{P_{2}}} \cdots\left(S_{M}\left(\nu_{M}\right)^{\dagger}\right)^{n_{P_{M}}} \mid \phi>
$$

where $S_{i}\left(\nu_{i}\right)^{\dagger}=a_{i+A}^{\dagger} \sigma_{i}^{\nu_{i}}$, with $\nu_{i}=0, \pm, z$. These states have eigenenergies given by

$$
E_{3}=E_{0}+\left(n_{1}+n_{2}+\cdots+n_{M}\right) .
$$

Clearly, excited energy levels are highly degenerate. However, we are still unable to develop a systematic rule to characterize the pattern of the degeneracy and to explain it with symmetries of the system (presumably, Yangian symmetry). 
In the limiting case where $M=M_{\uparrow}+M_{\downarrow}=0$, one has two types of bosons on the chain, the local electron pairs and the holes. The wavefunction $\phi$ becomes

$$
\phi\left(x_{1}, x_{2}, \cdots, x_{A} \mid z_{1}, z_{2}, \cdots, z_{Q}\right)=1
$$

which is obviously the ground state of the Hamiltonian $H$, with the ground state energy $E_{0}=-L(L-1) / 4$. With the raising operators, one may construct the first excited state quite easily:

$$
\phi^{\prime}\left(x_{1}, x_{2}, \cdots, x_{A} \mid z_{1}, z_{2}, \cdots, z_{Q}\right)=\left(x_{1}+x_{2}+\cdots+x_{A}\right) .
$$

The wavefunctions of higher excited states may be obtained by acting this wavefunction with raising operators again.

Besides this extended Hubbard model of long range type defined on a non-uniform lattice, we would like to introduce another model of extended Hubbard model of long range type. Consider a uniform lattice of sites equal-spaced. The size of the lattice is $L$. The Hamiltonian of the system is given by

$$
H=-\frac{1}{2} \sum_{1 \leq i \neq j \leq L} d(i-j)^{-2} \Pi_{i j},
$$

where the function $d(i-j)=\frac{L}{\pi} \sin (\pi(i-j) / L)$, and the permutation operator $\Pi_{i j}$ is the same as Eq. (2)). With our previous experience, one can simply write the first quantized Hamiltonian as follows:

$$
H=-\frac{1}{2} \sum_{1 \leq i \neq j \leq L} 1 / d^{2}\left(q_{i}-q_{j}\right) M_{i j} .
$$

It is very easy to prove that this extended Hubbard model is also integrable, once the Hamiltonian is written this way by using the relation of Fowler and Minahan [16]:

$$
\begin{aligned}
& L_{n}=\sum_{i=1}^{L} \pi_{i}^{n}, \pi_{i}=\sum_{j(\neq i)=1}^{L} z_{j} /\left(z_{i}-z_{j}\right) M_{i j}, \\
& {\left[L_{n}, L_{m}\right]=0,\left[L_{n}, H\right]=0 ; n, m=1,2, \cdots,}
\end{aligned}
$$

where $z_{j}=e^{2 \pi i q_{j} / L}, j=1,2, \cdots, L$. This thus provides a proof for the integrability of the extended Hubbard model. In the limiting case of no electron pairs, this system reduces 
to the long range t-J model [13 [15,8]. If there are no holes and no electron pairs, the Hamiltonian reduces to the Haldane-Shastry spin chain [11,12]. In the general case, to write the conserved quantities of the system in terms of second quantization, one may simply use the permutation symmetries of the wavefunction. In this paper, we shall not present the wavefunctions and the eigenenergy spectrum of this system. More results on this system will be given elsewhere.

In summary, we have introduced two integrable models of strongly correlated electrons, one defined on a non-uniform lattice, the other on a uniform lattice. The systems belong to the Jastrow-integrable-type. Particularly, the system on the non-uniform lattice has equalspacing energy spectrum. Because of the degrees of freedom of the paired electrons, the energy levels will have much larger degeneracy than the long range t-J model. One of the most interesting things is to determine the underlying symmetries explicitly that account for the degeneracy of each energy level.

I wish to thank V. E. Korepin for interesting conversations. Discussions with Mo-lin Ge and C. Gruber are acknowledged gratefully. This work was supported by the Swiss National Science Foundation. 


\section{REFERENCES}

[1] P. W. Anderson, Science 235, 1196 (1987).

[2] Lu Yu, Zhao-bin Su and Yan-min Li, Chinese J. of Physics 31 (Taipei), 579 (1993), references therein.

[3] E. H. Lieb and F. Y. Wu, Phys. Rev. Lett. 20, 1445 (1968), C. N. Yang, Phys. Rev. Lett. 19, 1312 (1967).

[4] C. N. Yang, Phys. Rev. Lett. 63, 2144 (1989).

[5] C. N. Yang and S. C. Zhang, Mod. Phys. Lett. B4, 759 (1990).

[6] F. H. L. Ebler, V. E. Korepin and K. Schoutens, Nucl. Phys. B 384, 431 (1992).

[7] F. H. L. Ebler, V. E. Korepin and K. Schoutens, Phys. Rev. Lett. 68, 2960 (1993).

[8] D. F. Wang and C. Gruber, Phys. Rev. B 49, 15712 (1994).

[9] A. P. Polychronakos, Phys. Rev. Lett. 70, 2329 (1993).

[10] C. Gruber and D. F. Wang, Phys. Rev. B 50, 3103 (1994).

[11] F. D. M. Haldane, Phys. Rev. Lett. 60, 635 (1988).

[12] B. S. Shastry, Phys. Rev. Lett. 60, 639 (1988).

[13] Y. Kuramoto and H. Yokoyama, Phys. Rev. Lett. 67, 1338 (1991).

[14] N. Kawakami, Phys. Rev. B 46, 1005 (1992).

[15] D. F. Wang, James T. Liu and P. Coleman, Phys. Rev. B 46, 6639 (1992).

[16] M. Fowler and J. A. Minahan, Phys. Rev. Lett. 70, 2325 (1993). 